\title{
The visualisation and quantification of human gastrointestinal fat distribution with MRI: a randomised study in healthy subjects
}

\author{
Dian Liu ${ }^{1}$, Helen L. Parker ${ }^{2}$, Jelena Curcic ${ }^{1,2}$, Werner Schwizer ${ }^{2}$, Michael Fried $^{2}$, Sebastian Kozerke ${ }^{1}$ and \\ Andreas Steingoetter ${ }^{1,2 *}$ \\ ${ }^{1}$ Institute for Biomedical Engineering, University and ETH Zurich, Gloriastrasse 35, 8092 Zurich, Switzerland \\ ${ }^{2}$ Division of Gastroenterology and Hepatology, University Hospital Zurich, Raemisstrasse 100, 8091 Zurich, Switzerland
}

(Submitted 2 September 2015 - Final revision received 24 November 2015 - Accepted 3 December 2015)

\begin{abstract}
We aimed to study the fate of fat during digestion. For this purpose, we validated and investigated the non-invasive quantification of gastric and duodenal fat emptying and emulsion processing (creaming and phase separation) using the MRI method iterative decomposition with echo asymmetry and least squares estimation (IDEAL). In total, twelve healthy subjects were studied on two separate visits in a single-blind, randomised, cross-over design study. IDEAL was utilised to repeatedly acquire quantitative fat fraction maps of the gastrointestinal tract after infusion of one of two fat emulsions: $\mathrm{E} 1$ (acid stable, droplet size $0.33 \mathrm{~mm}$ ) and $\mathrm{E} 4$ (acid unstable, $0.38 \mathrm{~mm}$ ). In vitro and in vivo validation was carried out using diluted emulsion and gastric content samples, respectively, and resulted in Lin's concordance correlation coefficients of 1.00 (95\% CI 0.98, 1.00) and 0.91 (95\% CI 0.87, 0.94), respectively. Fat fraction maps and intragastric emulsion profiles enabled the identification of features of intraluminal phase separation and creaming that were not visible in conventional MRI. Gastric fat emptying was faster for E4 compared with E1 with a difference of $2 \cdot 5(95 \%$ CI 1.9, 3.1) $\mathrm{ml} / \mathrm{h}$. Duodenal content volumes were larger for E1 than for E4 with a difference of $4.9(95 \%$ CI $3.9,8.5) \mathrm{ml}$. This study demonstrated that with IDEAL it was possible (1) to visualise the intragastric and duodenal fat distribution and (2) to quantify the differences in emptying, phase separation and creaming of an acid-stable and an acid-unstable emulsion. This method has potential to bridge the gap between current in vitro digestive models and in vivo behaviour and to be applied in the development of effective functional foods.
\end{abstract}

Key words: MRI: Water-fat separation: Lipid emulsions: Biophysics of fat digestion: Gastric physiology: Gastrointestinal function: Healthy subjects

In recent years, there has been a substantial increase in the number of reported nutrition-related diseases ${ }^{(1,2)}$. Further, obesity - which is a major risk factor for these diseases - has become a global problem ${ }^{(3)}$. Diets that are high in fat have often been cited as a leading cause for obesity ${ }^{(4)}$. However, dietary fat remains an important source of essential fatty acids and plays a major role in the absorption of nutrients and pharmaceuticals ${ }^{(5-7)}$. Therefore, this has prompted an increase in research efforts to understand how food, particularly fat, is processed and digested within the gastrointestinal (GI) $\operatorname{tract}^{(8)}$. The physical state of fat has been demonstrated to affect the rate of fat digestion ${ }^{(9,10)}$. Using fat emulsions, the in vivo physical state of fat can be adapted by careful design ${ }^{(11)}$. Therefore, there is keen interest from both physiological and product engineering perspectives in order to gain advanced insight into how fat is transformed during digestion and absorption $^{(6,12)}$.
To date, there is a reasonable correlation between in vitro models and in vivo outcomes of fat nutrient sensing ${ }^{(7,13-16)}$. These models together with extensive in vitro studies have generated a good understanding of how a controlled behaviour of fat within the GI tract can influence both nutrient sensing and uptake in vivo ${ }^{(17,18)}$. However, a complete and direct in vitroin vivo correlation is often hindered by the limited understanding of how intraluminal processes such as emptying dynamics, trituration and inter-mixing with secretions impact the structure of ingested fat emulsions. Therefore, a reliable and convenient method for the visualisation of fat within the human GI tract is required. MRI is a suitable technique meeting these requirements. MRI methods have been validated for both physiological and clinical measurements of GI function in health and disease states ${ }^{(19,20)}$. Moreover, a small number of studies have demonstrated the feasibility of MRI to visualise the structural changes of lipid emulsions and their impact on gastric

Abbreviations: FCV, fat content volume; GI, gastrointestinal; IDEAL, iterative decomposition with echo asymmetry and least squares estimation; TCV, total content volumes.

* Corresponding author: A. Steingoetter, email steingoetter@biomed.ee.ethz.ch 
Table 1. Composition and physical properties of the two fat emulsions

\begin{tabular}{lcc}
\hline & E1 & E4 \\
\hline Fat fraction & 20 wt\% (liquid) & 20 wt\% (liquid) \\
Emulsifier & 0.8 wt\% polysorbate 80 & 1 wt\% sodium caseinate \\
& & 0.25 wt\% monoglyceride \\
Mean particle size $(\mathrm{D} 4,3 \mu \mathrm{m})$ & 0.33 & 0.38 \\
Acid stable & Yes & No \\
Redispersible & Yes & Yes \\
\hline
\end{tabular}

emptying and satiation ${ }^{(11,21-23)}$. Further understanding can be provided by using quantitative MRI methods, which have been acknowledged to be of importance for food science and engineering ${ }^{(24-27)}$. Indeed, relaxation mapping methods have already been utilised for the quantification of gastric secretion $^{(28-31)}$. The water-fat separation method, iterative decomposition with echo asymmetry and least squares estimation (IDEAL) allows for a robust separation of the MR signal into its water and fat components ${ }^{(32-34)}$. Therefore, the IDEAL method can provide the visualisation and quantification of spatial distribution of water and fat in vivo ${ }^{(35)}$. This enables fat fraction maps to be extracted - that is, images with quantitative information - contrasting the commonly applied qualitative fat-selective imaging strategies. This technique has not been applied to the GI tract so far, and thus is a potentially promising tool to study the spatial distribution and emptying dynamics of total intraluminal content alongside an independent measure of fat. This knowledge may ultimately offer deeper insights into the biophysics of gastric mixing and fat digestion. This in turn may provide the means to further bridge the gap between in vitro models and in vivo studies.

Therefore, the overarching aim of this study was to validate the non-invasive quantification of fat fraction in gastric and duodenal contents by MRI, utilising the water-fat separation method IDEAL. To this end, two lipid emulsions with differing acid stabilities were utilised in order to quantify and assess the dynamic processing of fat within the stomach (phase separation and creaming) and duodenum in a randomised, cross-over, single-blinded study design.

\section{Methods}

\section{Fat emulsions}

Two types of previously described and tested fat emulsions E1 and $\mathrm{E} 4$ were used in this study. The composition and properties of the emulsions are outlined in Table $1^{(11)}$; they were prepared as described in online Supplementary Appendix S1.

\section{Fat fraction maps}

In order to compute fat fractions based on chemical shift-induced signal variations, an accurate fat model of rapeseed oil was required. Therefore, a spectral multi-peak fat model of rapeseed oil was generated to quantify the fat peaks using a spectroscopic MR measurement on a 3-Tesla MRI scanner (Ingenia, Philips
Healthcare). This fat model was used in the IDEAL reconstruction of all fat fraction maps (online Supplementary Fig. S1).

All MRI data were acquired using a $1 \cdot 5$-Tesla MRI scanner (Achieva, Philips Healthcare), using an abdominal phased array coil (4-channel SENSE body coil; Philips Healthcare) with four elements. In order to acquire the fat fraction maps, IDEAL scans were performed with a multi-point echo sequence. The scanned data were then reconstructed by combining the multiple images acquired at the different echo times using the IDEAL approach $^{(35)}$. In reconstruction, B0 field inhomogeneity, T2* decay and the multi-peak spectral fat model were incorporated. After separating the data into water and fat images using the IDEAL reconstruction, the resulting fat fraction maps were obtained by calculating the ratio of the water and fat images.

\section{Study design}

Study population. This study was conducted according to the guidelines laid down in the Declaration of Helsinki, and all the procedures involving human subjects were approved by the Ethics Committee of the Canton of Zurich (KEK-ZH-Nr. 2014-0220); the trial was registered at ClinicalTrials.gov with identifier NCT02226029. Written informed consent was obtained from all subjects.

The present study was performed using a randomised, singleblinded, two-armed, cross-over study design. A total of twelve healthy subjects were recruited by advertisement. At an initial screening visit, subject eligibility criteria were assessed. Therefore, those invited to participate had neither current health problems nor a history of GI disease or abdominal surgery (excluding appendectomy or hernia repair). Subjects were also required to provide a negative pregnancy test (if applicable), have a BMI between 18 and $25 \mathrm{~kg} / \mathrm{m}^{2}$, be a non-regular smoker, aged between 18 and 50 years and have no contraindication to MRI. All the participants underwent MRI scans of $3 \mathrm{~h}$ on two different study days. During each study day, subjects were given one of the two emulsions. Assuming a SD of $1.5 \%$ based on earlier data, we estimated that a total of twelve subjects would be needed to detect a difference of $2 \%$ fat fraction, with a defined power of 0.9 . Two computer-generated, permutated blocks of twelve defined the randomisation sequence for all subjects. Blinding of the subjects was ensured, as the emulsions had the same visual appearance.

Measurements. On the study day, subjects arrived in the morning at the MR centre at the University Hospital Zurich after 


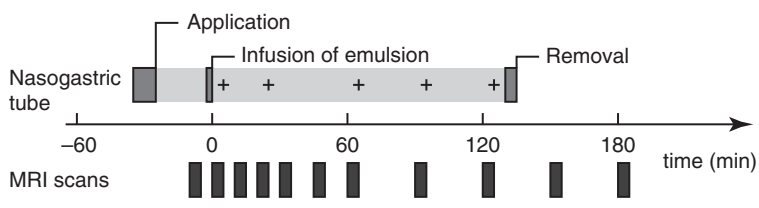

Fig. 1. Timeline of the study protocol. Exemplary gastric sampling time points are indicated by crosses.

$8 \mathrm{~h}$ of fasting. A silicone nasogastric tube (Freka $\mathrm{CH}$ 12; Fresenius Kabi) was positioned in the gastric corpus through the nostril of the subject. The subjects were infused with $200 \mathrm{ml}$ $(1590 \mathrm{~kJ}(380 \mathrm{kcal}))$ of the emulsions within $1 \mathrm{~min}$ in order to control for the effect of inter-individual tolerance to texture and taste. End of infusion was taken as starting point $t=0 \mathrm{~min}$ of gastric emptying. Subjects were positioned into the right decubitus in the MR scanner and were allowed to assume a sitting position outside the scanner during longer scan pauses. In these positions, intragastric gas accumulates in the fundus, which ensures a maintained rate of gastric emptying and distribution despite changes in body position ${ }^{(36)}$. The first MRI was acquired before meal infusion in order to obtain the volume of residual stomach content in the fasted state. All other MRI were acquired within scan blocks, which were performed at time points $t=0,10,20,30,45,60,90,120,150$ and $180 \mathrm{~min}$, yielding ten blocks in total. Scans were performed during one breath-hold each, lasting from 5 to $34 \mathrm{~s}$. Fig. 1 shows the timeline of the study day.

Up to five samples of gastric content of $2 \mathrm{ml}$ each were collected via the nasogastric tube between $t=0$ and $120 \mathrm{~min}$. The aspirated gastric samples (online Supplementary Fig. S2) were used to obtain laboratory fat fractions in order to validate the fat fractions obtained using the MRI method IDEAL. After $t=120 \mathrm{~min}$, the nasogastric tube was removed.

In each MRI scan block, four image types were acquired: IDEAL and volume scans were used for obtaining gastric and duodenal content volumes. Fat-selective scans yielded qualitative fat-selective images, which served as a comparator to the quantitative fat fraction maps. High-resolution volume scans were acquired in order to determine the position of the nasogastric tube (online Supplementary Appendix S2).

\section{Fat fraction validation}

In vitro. An in vitro validation of the fat fraction measurements obtained by IDEAL was performed. The emulsions were diluted to give a series of nine fat fraction samples between 0 and $20 \%$. The samples were placed into plastic Falcon ${ }^{\circledR}$ tubes (Corning) and then simultaneously positioned in the MRI scanner to be measured by IDEAL. Mean fat fraction values were calculated within a region of interest $(\mathrm{ROI})$ of $5 \times 5$ voxels for each sample and compared with the nominal fat fraction values by Lin's concordance correlation coefficient $r_{c}^{(37)}$.

In vivo. The fat fraction measurements provided by IDEAL were validated in vivo with nasogastric aspirates ${ }^{(38)}$. The tip of the catheter was first identified in the high-resolution volume scan, and then a ROI of $5 \times 5$ voxels was chosen at the

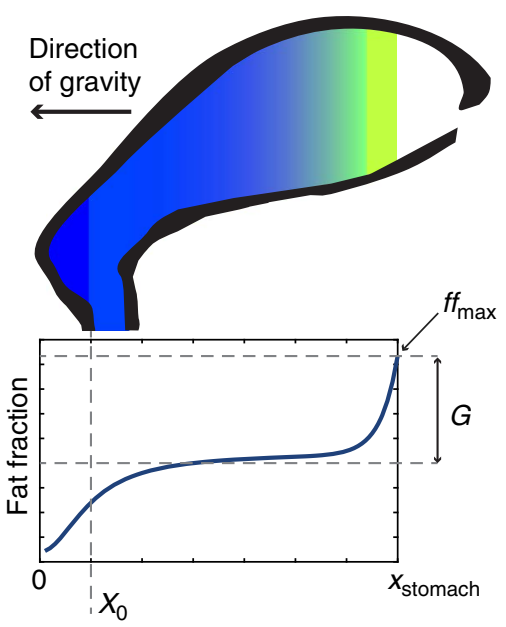

Fig. 2. Modelling of fat layering. Schematic to visualise the computed emulsion profiles and the applied model.

appropriate position in the corresponding fat fraction map (online Supplementary Fig. S3). The fat fraction of the gastric samples was measured by gravimetric analysis. Each sample was first centrifuged at $4300 \boldsymbol{g}$ for $5 \mathrm{~min}$ (Labofuge 400R; Heraeus Instruments) to enhance the phase separation between the emulsion and gastric juice. If possible, the clear gastric juice part was extracted from the gastric sample in order to minimise the contribution of residual particles to the resulting fat fractions. The weights of all samples and extracted gastric juice parts were determined. Subsequently, the samples were dried in a controlled environment and the weights of all samples were measured again after complete evaporation. Gastric samples that either developed mould during the drying process, contained solid particles or could not be identified in the MRI because of ambiguous detection of the nasogastric tip were excluded from the analysis. Laboratory fat fractions were calculated by obtaining the corresponding weight ratios. IDEAL and laboratory fat fractions were compared against each other by $r_{c}$.

\section{Data analysis}

Fat distribution and emptying. A custom software tool written in MATLAB 2012b (The MathWorks) was used for image segmentation and related volume calculation. Gastric or duodenal total content volumes (TCV), which are defined as content volumes without intraluminal air, were derived by semi-automatic segmenting of the content contours of each volume image ${ }^{(39)}$.

Gastric or duodenal fat contents were obtained by applying the contours of the volume images to the fat fraction maps. Fat content volume (FCV) was calculated at each time point by weighting TCV with its corresponding average fat fraction value obtained from the fat fraction maps. Gastric and duodenal TCV and their respective FCV were plotted over time to generate volume emptying curves.

Intragastric emulsion profiles. In order to assess creaming and phase separation of the ingested emulsions, intragastric fat fractions were averaged at each level along the direction of 
(a)

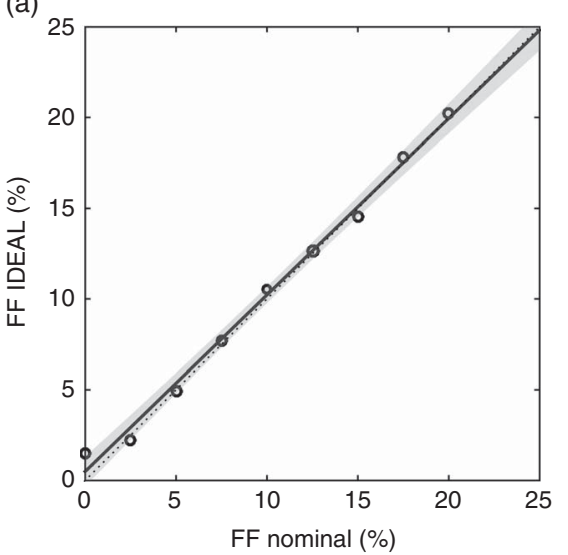

(b)

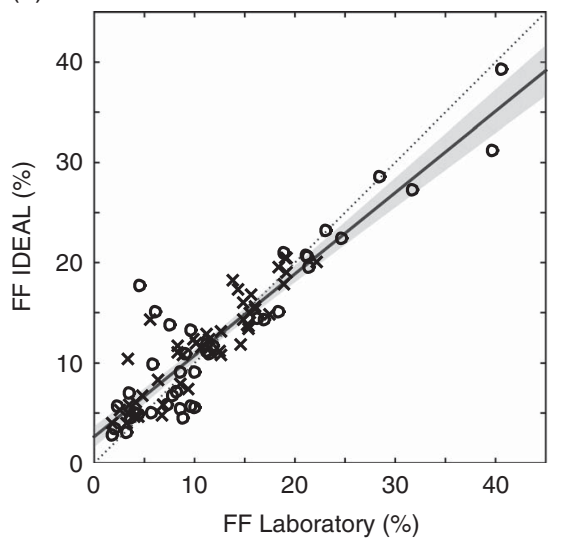

Fig. 3. In vitro and in vivo fat fraction (FF) validation. (a) Correlation plot showing the agreement between the in vitro experiment with iterative decomposition with echo asymmetry and least squares estimation (IDEAL) fat fractions and the nominal fat fractions. (b) Correlation plot showing the agreement between in vivo experiment with laboratory fat fractions and IDEAL fat fractions including data from E1 $(\mathrm{X})$ and E4 $(\mathrm{O})$. Each plot depicts the line of identity (..........) and the linear regression fit (—) with $95 \% \mathrm{Cl}($ ).

gravity as illustrated in Fig. 2. Using these intragastric emulsion profile plots, the time point of maximum phase separation was defined by the occurrence of a maximum fat fraction $\left(f f_{\max }\right)$ in the stomach. A data-driven bi-phasic model (online Supplementary Appendix S3) was fitted to these profiles in order to detect (1) the location of phase separation in the distal stomach $\left(x_{0}\right)$, (2) the normalised extent of this phase separation $E=x_{0} /$ $x_{\text {stomach }}$ and (3) the increase or gradient in the fat fraction towards the proximal stomach $G$ (see again Fig. 2). These parameters may be considered as surrogate markers for the degree of phase separation and creaming, respectively. The non-linear least-squares fit was performed in MATLAB 2012b.

Statistics. Statistical analyses were carried out using programme R, version $3.1 .3^{(40)}$. The effects on fat emptying and emulsion profile were tested by linear mixed-effects models using function lmer of $\mathrm{R}$ package lme4 (version 1.1-7). The outcome measures TCV, FCV and fat fraction were modelled with subject as a random effect. For the stomach, emulsion, time and fasted content volume (TCV-1) were used as fixed effects, whereas only emulsion was used as a fixed effect for the duodenum. The outcome measures $f f_{\max }, E$ and $G$ were modelled with subject as a random effect and emulsion as a fixed effect. Model parameter estimates are presented with $95 \%$ CI.

\section{Results}

In total twelve subjects were included in the study for MRI measurements. MRI scans were successfully performed on all the twelve subjects (five men, seven women; mean age: $22 \cdot 0$ ( $\mathrm{sD} 2.0$ ) years; mean BMI: $21.9(\mathrm{sD} 1.5) \mathrm{kg} / \mathrm{m}^{2}$ ). Gastric image data of three scan blocks and duodenal image data of seven scan blocks were missing because of technical errors. Nevertheless, gastric and duodenal volume curves were successfully determined in all subjects. The nasogastric tube, the emulsions and the breath-holds were well tolerated by all subjects.

\section{Fat fraction validation}

Fig. 3(a) depicts the scatterplot of the in vitro MRI measurements (nine data points), which demonstrates the agreement between nominal fat fractions and fat fractions measured by IDEAL. Lin's concordance correlation coefficient was 1.00 (95\% CI 0.98, 1.00).

The laboratory fat fraction values from gastric content samples were determined from a total of eighty-eight included samples. In total twenty-seven samples were excluded from the analysis; Lin's concordance correlation coefficient was 0.91 (95\% CI 0.87, 0.94). The agreement between the two measurement methods is presented in the scatterplot of Fig. 3(b).

\section{Fat distribution and emptying}

Statistical analyses of emulsion profiles were performed only on those during the time point of maximum phase separation (twenty-four data points). Statistical analyses on emptying were performed on 238 gastric and 233 duodenal observations.

Stomach. The different intragastric stability of the fat emulsions resulted in different structuring of intraluminal content. In the standard qualitative fat-selective images, this was indicated by changes in MR signal intensities, where higher fat contents resulted in brighter signals. Colour coding was applied for the fat fraction maps, where blue and red indicated 0 and $100 \%$ fat fraction, respectively. A direct comparison between the two image types showed that they were in agreement with each other, both depicting the different dynamics of phase layering and revealing the different dynamics of fat structuring (Fig. 4). However, inhomogeneity features such as fat fraction gradients and fat pockets could hardly be identified in the fat-selective images but were obvious in the fat fraction maps.

Typical fat distributions in the stomach of the acid-stable emulsion E1 and the acid-unstable emulsion E4 are depicted in the fat fraction maps in Fig. 5(a). E1 largely maintained its stability throughout the 180 -min scanning period. This is

\footnotetext{
and the breath-holds were well tolerated by all subjects.
} 


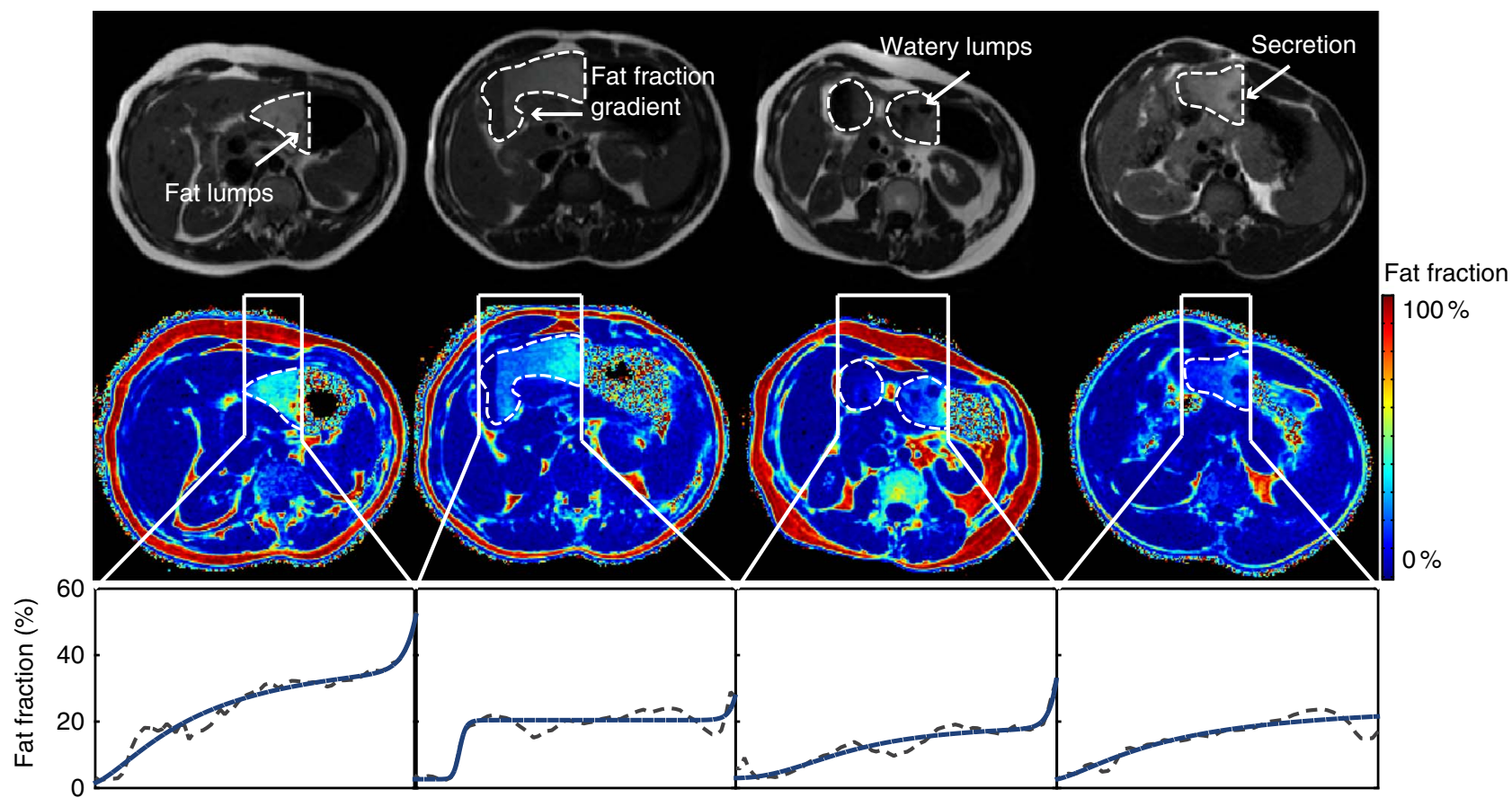

Fig. 4. Gastric content of E4 of four different subjects within the first $60 \mathrm{~min}$. The qualitative fat-selective images (upper panel), the corresponding colour-coded fat fraction images (middle panel) and emulsion profiles (lower panel) show the inter-individual variations in intragastric fat distributions. The dashed white curves outline the, intraluminal content. Although both image types are in good agreement with each other, features such as fat lumps or fat fraction gradients are less evident in the first image type. The measured (----) and fitted (_) emulsion profiles reflect the differing extents of phase separation and creaming.

demonstrated by the gradual transition of the homogeneous gastric content to the deeper shades of blue, which represent lower fat fractions. Further, this indicates that gastric secretion was continuously released into the stomach and then mixed with gastric content. E4 underwent clear structural changes under gastric conditions, resulting in less homogeneous intragastric fat distributions. Shortly after meal infusion, E4 exhibited creaming, flocculation of fat and separation into two phases. This phase separation occurred as a region of higher fat content developing on top of the remaining gastric content. This bi-phasic fat distribution led to a faster emptying of the lower fat content phase present in the distal stomach. In a few subjects, re-emulsification of the flocculated fat could be observed in the image data.

These observations are nicely reflected in the emulsion profiles. The dynamics of phase separation, including the degree of flocculation and subsequent re-emulsification, varied considerably between subjects, as can be observed in the image series of Fig. 4.

Fig. 5(a) shows the change in the intragastric fat fraction over time in one subject. Both the fat fraction maps and the emulsion profiles nicely reflected the different properties of E1 and E4. Individual emulsion profiles of $\mathrm{E} 4$ revealed large differences between the subjects as visualised in Fig. 4. No creaming was observed for E1 with $f f_{\max }$ of 21.6 (95\% CI 16.4, 26.4) \% and $G$ of $0 \cdot 1(95 \% \mathrm{CI}-0 \cdot 1,0 \cdot 2) \% / \mathrm{mm}$. Creaming was stronger in E4 compared with E1, where $f f_{\text {max }}$ was larger by $21.2(95 \%$ CI $14 \cdot 8$, $28 \cdot 1) \%$ and $G$ was larger by $0.2(95 \%$ CI $0.04,0.3) \% / \mathrm{mm}$. The observed highest degree of creaming - that is, the upper range of $f f_{\max }-$ was $68 \%$ in $\mathrm{E} 4$. The extent of phase separation E for E1 was small with $0.1(95 \% \mathrm{CI} 0 \cdot 0,0.3)$; E4 showed values of $\mathrm{E}$ that did not differ compared with E1 (difference: $0 \cdot 1$ (95\% CI 0.0, 0.3)).
The different structuring of intraluminal content described above resulted in different emptying patterns of E1 and E4. Individual gastric emptying curves of TCV and FCV grouped per fat emulsion are presented in Fig. 6. Initial TCV - that is, the content volume directly after meal intake - showed large differences between subjects. This was attributed to the effect of TCV-1 with a difference of $0.9(95 \% \mathrm{CI} 0.7,1.2) \mathrm{ml}$, rather than the effect of the emulsion type with a difference of $-2 \cdot 2(95 \% \mathrm{CI}-13 \cdot 6,9 \cdot 1) \mathrm{ml}$. Therefore, $1 \mathrm{ml}$ of TCV-1 caused an increase in initial TCV by $0.9 \mathrm{ml}$, which indicates that most residual gastric content still remained in the stomach after intake. TCV of the acid-stable E1 remained high until the end of the scanning period, supporting the observations for the intragastric fat distribution of continuous and intensive dilution with gastric secretion. In several cases, an increase in TCV was observed where the amount of released secretion exceeded that of gastric content emptying. In contrast, TCV of the acid-unstable E4 revealed a distinct non-linear content emptying pattern with rapid decreases during the scanning period. Consequently, TCV emptying for E1 (17 (95\% CI 12, 22) ml/h) was slower by $40(95 \%$ CI 33, 48) ml/h compared with $\mathrm{E} 4$.

Although gastric content emptying curves exhibited large variations among subjects, which were reflected in the large $\mathrm{CI}$ values of TCV, FCV curves of both emulsions decreased steadily and yielded smaller CI values. Fat emptying of E1 was 8.6 (95\% CI 9.5, $7 \cdot 7) \mathrm{ml} / \mathrm{h}$, which was slower by $2.5(95 \%$ CI $1.7,3 \cdot 3) \mathrm{ml} / \mathrm{h} \mathrm{com}-$ pared with E4.

Duodenum. Duodenal fat fractions were lower compared with gastric fat fractions. Similar to the gastric content 

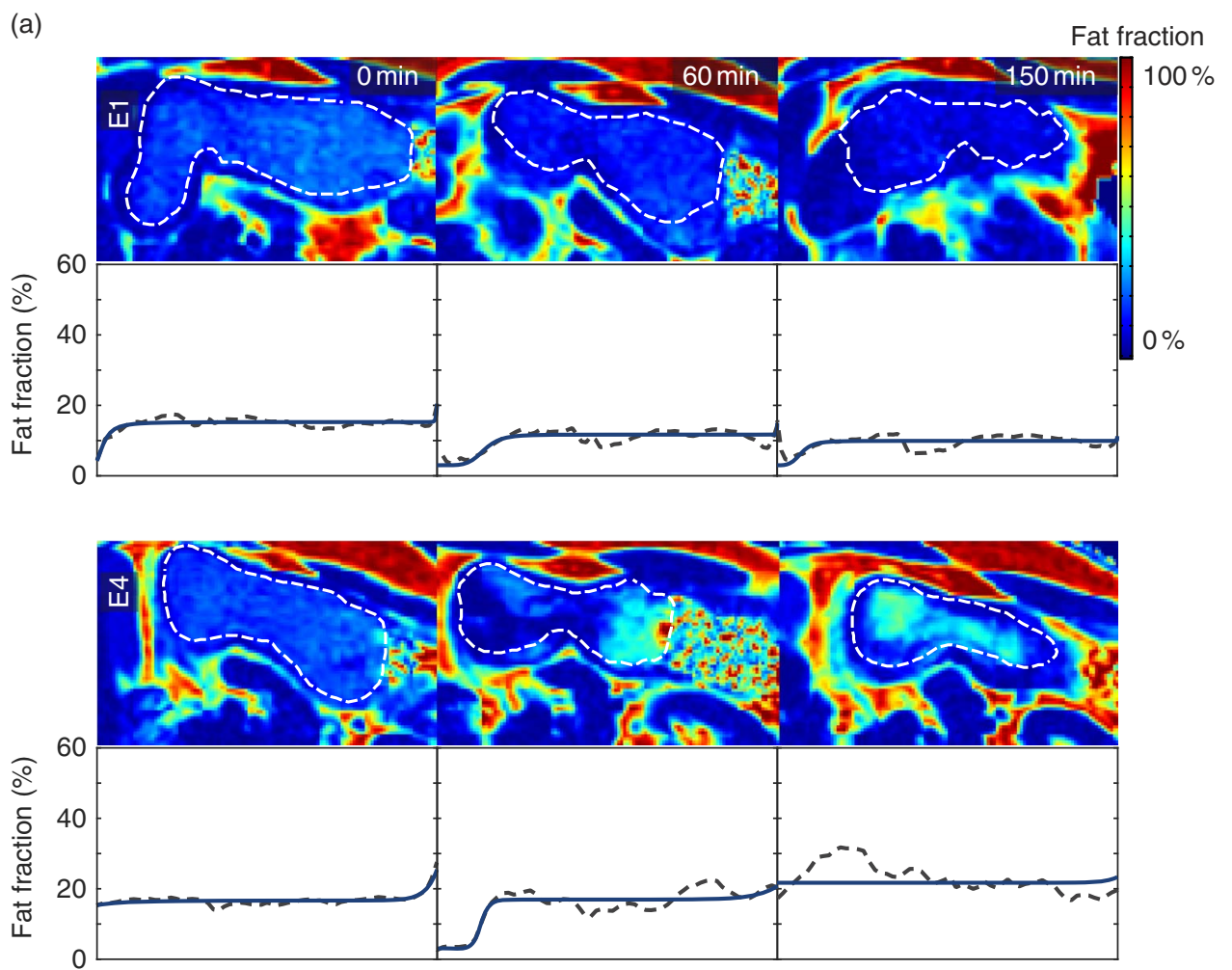

(b)

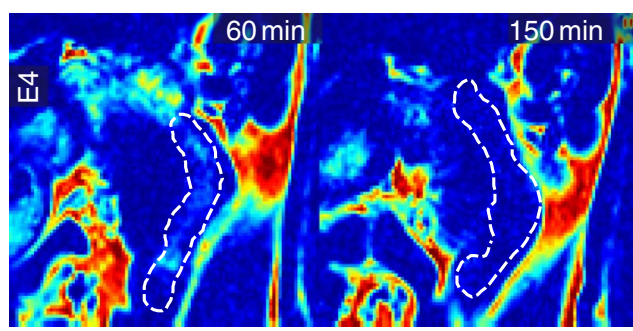

Fig. 5. Gastric and duodenal contents of one subject at different time points. (a) Images showing the cropped and magnified colour-coded gastric fat fraction maps of E1 (upper panel) and E4 (middle panel). The corresponding emulsion profile plots show the respective measured (--.-.) and fitted ( $(-)$ intragastric fat fraction profiles (lower panel). (b) Images showing the cropped and magnified colour-coded duodenal fat fraction maps of E4 (upper panel). E1 exhibited homogeneous fat distributions, and is therefore not shown. The dashed white curves outline the intraluminal content. It is to be noted that the whole stomach volume rather than a single slice was used for the calculation of the emulsion profiles. Therefore, they cannot be directly compared with the corresponding fat fraction maps.

distributions, duodenal contents for E1 appeared more homogeneous. For E4, the duodenum was irregularly flushed with portions of high fat content from the stomach, as depicted in Fig. 5(b).

Fig. 7 demonstrates the duodenal curves for the measures of TCV and FCV, which exhibited less structure than their gastric counterparts. Time did not significantly predict any of the three measures (TCV: 0.01 (95\% CI -0.01, 0.04) ml/h; FCV: 0.001 (95\% CI $-0.001,0.003) \mathrm{ml} / \mathrm{h}$; fat fraction: 0.0 (95\% CI -0.008 , $0.008) \%$, and was therefore removed as a fixed effect in subsequent models. After initial passage of the emulsion through the duodenum, duodenal fat fraction ranged between 5 and $10 \%$ for E1. Duodenal TCV for E1 was 22 (95\% CI 17, 27) $\mathrm{ml}$, which was larger by $6 \cdot 3(95 \%$ CI 3.0, 9.3) ml compared with E4. For duodenal FCV, E1 was 1.7 (95\% CI 1.4, 2.1) $\mathrm{ml}$ and also exhibited larger volumes compared with $\mathrm{E} 4$ with a difference of 0.5 (95\% CI $0.2,0.8) \mathrm{ml}$.

\section{Discussion}

This study has demonstrated that with IDEAL it was possible (1) to simultaneously visualise the intragastric and duodenal fat distribution and (2) to quantify the differences in emptying, phase separation and creaming of an acid-stable and acidunstable emulsion.

The IDEAL method was validated both in vitro and in vivo. The in vitro MR measurements showed an excellent linear dependency and agreement between nominal and IDEAL fat fractions. The in vivo validation measurements using the gastric aspirates, however, resulted in lower Lin's concordance, exhibiting larger and more varying differences. These differences were caused by the following systematic measurement biases. First, the weight of the emulsifiers was included in the final weight after drying. Experiments confirmed that this caused the slope of the regression line between laboratory and nominal fat 
(a)

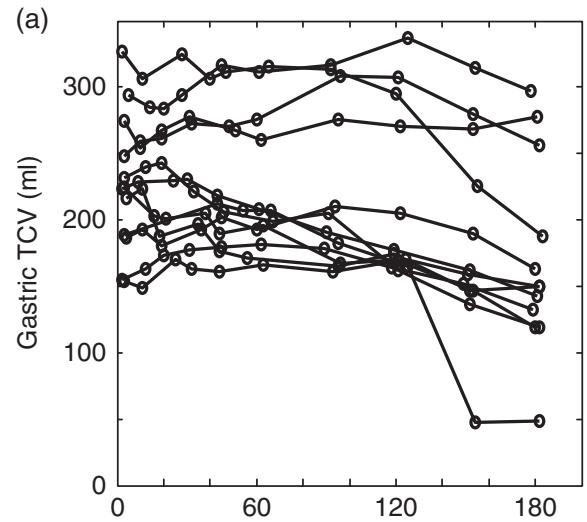

(c)

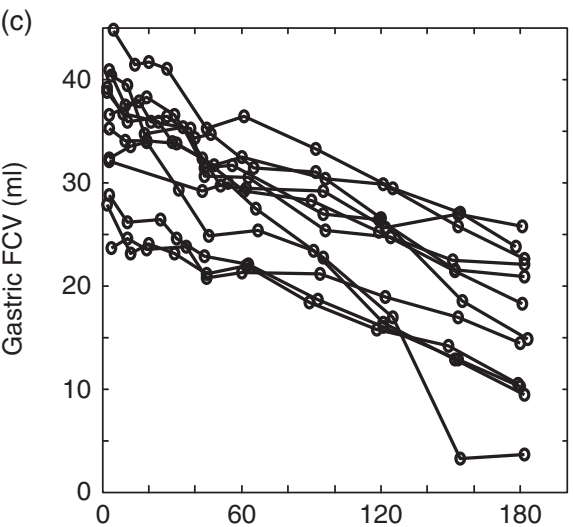

(b)

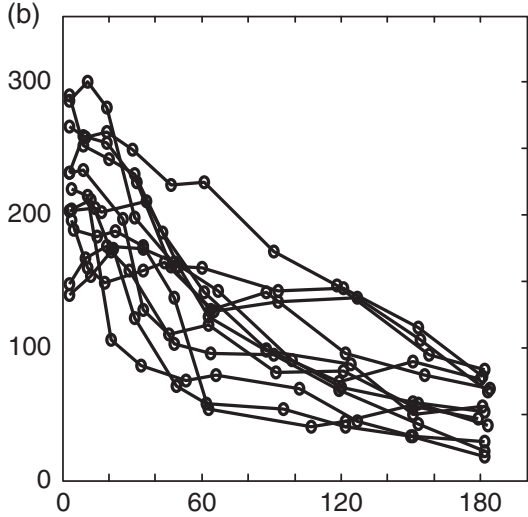

(d)

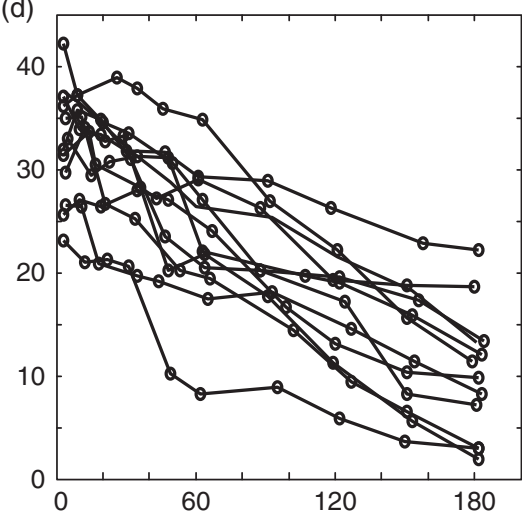

Fig. 6. Change of gastric total content volumes (TCV) and fat content volume (FCV) during emptying. Individual changes in gastric TCV (upper row) and FCV (lower row) over time are grouped by E1 (left column) and E4 (right column). Despite the large inter-individual differences and patterns in gastric TCV, resulting gastric FCV curves showed a somewhat steady fat emptying for both emulsions.

fractions to decrease by up to $10 \%$. Second, experiments further confirmed that gastric juice contained up to $2 \%$ of particles other than water, which also remained as residual weight after drying. At $0 \%$ fat fraction, this overestimated the measured value, therefore causing a positive intercept of the regression line. Finally, the large spread of residuals was due to the measurement procedure. Although only those samples with a clear identifiable catheter tip position were included in the analysis, little deviations from the true location of the sample within the stomach could lead to large measurement errors, especially in inhomogeneous regions or regions close to the gastric wall. Despite all these limitations, the detected in vivo correlation was higher than that previously reported for an inversion-recovery-based MRI quantification method ${ }^{(38)}$.

The different intragastric stability of the fat emulsions affected the distribution of the intraluminal content, which could be visualised with both fat-selective images and the quantitative fat fraction maps. The two image types revealed similar structuring of fat emulsion and were in general in good agreement with each other. However, the fat-selective images could not reveal all the features of inhomogeneity evident in the fat fraction maps. In contrast to fat-selective images, fat fraction maps allowed an assessment of the degree of phase separation and creaming and the resulting emptying rate of fat content. The stable fat emulsion always showed a homogeneous gastric fat distribution where fat was increasingly diluted with gastric secretion. The unstable fat emulsion showed highly variable gastric fat distributions with associated bi-phasic layering.

The differences in structuring of intragastric content caused by the different intragastric stability of the fat emulsions were also reflected in the emptying patterns. The stable fat emulsion exhibited very slow gastric content emptying among all subjects, whereas the unstable emulsion showed inter-individual bi-phasic emptying patterns with faster emptying of the separating phase of lower fat content. These observations correspond to findings reported from a previous study ${ }^{(11)}$ using the same fat emulsions. Large inter-individual differences were observed for the initial content volumes. However, these were attributed to the residual content volumes in the fasted stomach rather than to the emulsion type.

Owing to the steady emptying pattern of the fat in both emulsions, the emptying was approximated by a linear model. The fat-emptying rate of the unstable emulsion was faster than the stable emulsion by approximately $41 \%$. This may be due to a larger fat droplet size of the re-emulsified high-fat content phase compared with the artificially small original droplet sizes of the emulsions. Previous data have confirmed that a reduction in droplet size of fat emulsions delays gastric emptying due to more effective lipase activity and increased intestinal sensing and feedback ${ }^{(9)}$.

The model derived from the observed bi-phasic layering of intragastric content yielded a good fit to the emulsion profiles. 
(a)

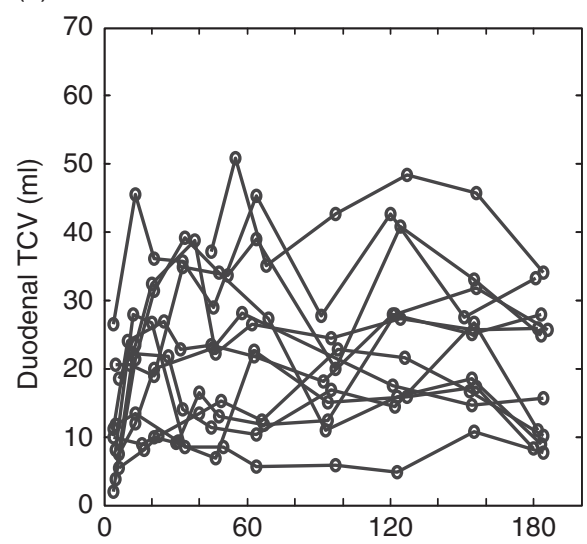

(c)

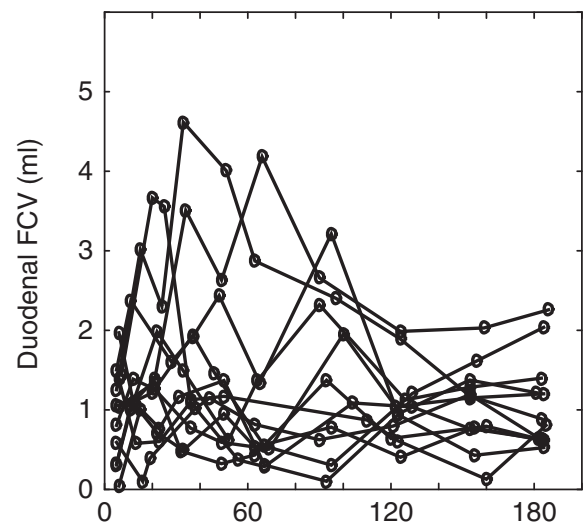

(b)

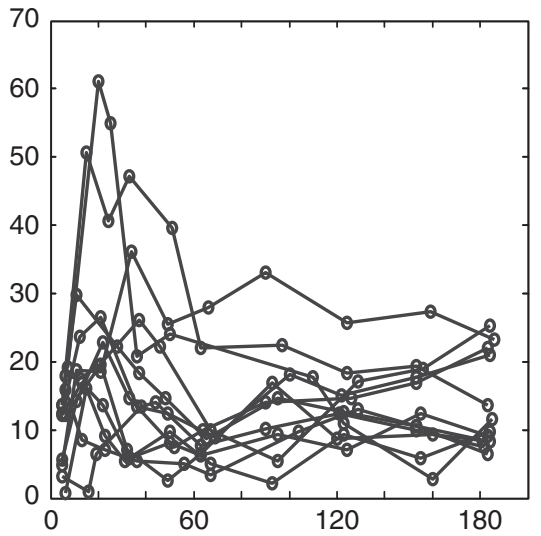

(d)

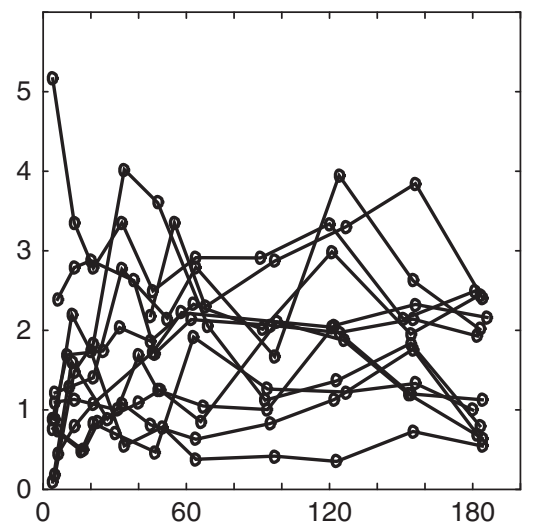

Fig. 7. Change of duodenal total content volumes (TCV) and fat content volume (FCV) during emptying. Individual changes in duodenal TCV (upper row) and FCV (lower row) over time are grouped by E1 (left column) and E4 (right column).

Although the degree of phase separation was not different between the emulsions, the acid-unstable E4 exhibited a strong degree of creaming. Maximum fat fraction values were twice as large and had a four times larger gradient. Moreover, large variations in emulsion profiles were observed in E4, indicating inter-individual differences in the composition and acidity of gastric secretion. The fluctuations in the emulsion profiles visible in many profiles may be indicators of flocculation of fat droplets, which affect gastric emptying and satiation as previously shown ${ }^{(11)}$. A thorough analysis on this topic was, however, beyond the scope of this study.

Duodenal emptying curves exhibited less structure than their gastric counterparts; a temporal effect of the curves could not be detected. Although the dynamics of gastric emptying are mainly influenced by content emptying into the duodenum on the one hand and release of gastric secretion on the other hand, duodenal emptying is affected by the incoming content from the stomach, emptying into the small intestine and release of bile. This additional complexity seems to be reflected in the duodenal volume curves that exhibited higher fluctuations than their associated gastric curves. The peaks in duodenal FCV may be partly attributed to changes in the subject's body position that occurred in between the scans. A sudden shift of high fat content towards the distal part of the stomach could have caused the duodenum to be flushed with high fat content. A thorough analysis on how body position affects duodenal fat volume was beyond the scope of this study.

Duodenal volumes of total content and fat were both larger for the stable emulsion - that is, by a factor of 34 and $30 \%$ respectively - whereas mean fat fractions remained at similar levels. Although the larger duodenal TCV for E1 indicates increased gallbladder secretion due to increased fat sensing, the larger FCV may be explained by a delayed fat transport due to a reduced number of duodenal pressure waves ${ }^{(9)}$.

Two fat emulsions of highly artificial composition were used in order to validate the quantitative MRI methodology. However, the application of IDEAL is not limited to these emulsion types. The application to other fat emulsions is straightforward by substituting the fat model acquired for the rapeseed oil with the appropriate fat model, offering the possibility to choose from a broad range of vegetable or dairy oils. Problems could, however, arise for solid meals or meals generating solid particles within the GI tract. Solid materials cause signal voids in the MR images, resulting in undetectable intraluminal contents.

A major constraint of this method is the rather long maximum breath-hold time used in the study protocol of $34 \mathrm{~s}$, which currently limits the application to healthy individuals. In the 
future, under-sampling strategies to accelerate data acquisition will reduce scan duration per breath-hold, enabling studies in patients as well ${ }^{(41)}$. Instead of reducing the breath-hold time, accelerated data acquisition could also be utilised to cover larger fields of view - for example, subjects with larger waist circumferences - and for simultaneous acquisition of gastric and intestinal data. Images covering the entire intestinal tract are useful to provide additional insights into food transit and fat digestion in the lower GI tract. The feasibility of fat quantification in these parts still needs to be investigated because of two factors: first, lower fat contents due to continuous dilution by gastric and duodenal juice, causing the fat fractions to fall below the limit of detection or accuracy, and, second, the disintegration of fat due to digestive enzymes, causing the fat model to be

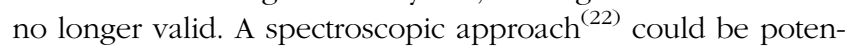
tially applied for analysing the fat model and its validation in the lower GI tract.

In conclusion, MRI was validated and applied for quantifying the in vivo processing of fat emulsions in the upper GI tract using the water-fat separation method IDEAL. This not only revealed information about the complexity of food structuring during digestion, but also gave insight into the fate of the fat component with regard to emptying and its characteristics under forces such as trituration and peristaltic activity. These methods have great potential to improve the in vitro-in vivo correlation of current digestive models and to be applied in the process of developing effective functional foods that control energy intake and homoeostasis. Both aspects are of key importance in the field of nutrition-related healthcare and eating disorder treatment.

\section{Acknowledgements}

The authors thank Tim J. Wooster for critically reviewing the manuscript and providing essential materials and knowledge for the emulsion formulation and production, Nathalie Scheuble for providing support for in vitro analyses, Martina Bertschinger for her help in emulsion production and supporting medical procedures during the pilot study period and, finally, Vlad Ceregan for assisting during study days.

This work was supported by the Swiss National Science Foundation (SNSF), grant numbers 146409 and 145141. The SNSF had no role in the design, analysis or writing of this article.

D. L., W. S., M. F., S. K. and A. S. designed research; D. L., H. L. P. and J. C. conducted research; H. L. P. screened and recruited subjects; D. L. analysed the data; D. L. and A. S. performed the statistical analyses; D. L., H. L. P., J. C. and A. S. wrote the paper; D. L., W. S., M. F. and A. S. had primary responsibility for final content.

The authors declare that there are no conflicts of interest.

\section{Supplementary material}

For supplementary material/s referred to in this article, please visit http://dx.doi.org/10.1017/S0007114515005188

\section{References}

1. Zimmet P, Alberti KG \& Shaw J (2001) Global and societal implications of the diabetes epidemic. Nature 414, 782-787.

2. Reddy KS (2002) Cardiovascular diseases in the developing countries: dimensions, determinants, dynamics and directions for public health action. Public Health Nutr 5, 231-237.

3. Poirier P, Giles TD, Bray GA, et al. (2006) Obesity and cardiovascular disease: pathophysiology, evaluation, and effect of weight loss: an update of the 1997 American Heart Association Scientific Statement on Obesity and Heart Disease from the Obesity Committee of the Council on Nutrition, Physical Activity, and Metabolism. Circulation 113, 898-918.

4. Chan M (2013) WHO Director-General addresses health promotion conference. Opening Address at the 8th Global Conference on Health Promotion. 10 June 2013, Helsinki, Finland.

5. Porter CJ, Trevaskis NL \& Charman WN (2007) Lipids and lipid-based formulations: optimizing the oral delivery of lipophilic drugs. Nat Rev Drug Discov 6, 231-248.

6. Fave G, Coste TC \& Armand M (2004) Physicochemical properties of lipids: new strategies to manage fatty acid bioavailability. Cell Mol Biol (Noisy-le-grand) 50, 815-831.

7. Carey MC, Small DM \& Bliss CM (1983) Lipid digestion and absorption. Annu Rev Physiol 45, 651-677.

8. Golding M \& Wooster TJ (2010) The influence of emulsion structure and stability on lipid digestion. Curr Opin Colloid Interface Sci 15, 90-101.

9. Seimon RV, Wooster T, Otto B, et al. (2009) The droplet size of intraduodenal fat emulsions influences antropyloroduodenal motility, hormone release, and appetite in healthy males. $\mathrm{Am} \mathrm{J}$ Clin Nutr 89, 1729-1736.

10. Hussein MO, Hoad CL, Stephenson MC, et al. (2015) Magnetic resonance spectroscopy measurements of intragastric fat fraction of oil emulsions in humans. Eur J Lipid Sci Tech 117, 31-36.

11. Steingoetter A, Radovic T, Buetikofer S, et al. (2015) Imaging gastric structuring of lipid emulsions and its effect on gastrointestinal function: a randomized trial in healthy subjects. $\mathrm{AmJ}$ Clin Nutr 101, 714-724.

12. McClements DJ, Decker EA \& Park Y (2009) Controlling lipid bioavailability through physicochemical and structural approaches. Crit Rev Food Sci Nutr 49, 48-67.

13. Wooster TJ, Day L, Xu M, et al. (2014) Impact of different biopolymer networks on the digestion of gastric structured emulsions. Food Hydrocolloids 36, 102-114.

14. Reis P, Raab T, Chuat J, et al. (2008) Influence of surfactants on lipase fat digestion in a model gastro-intestinal system. Food Biophys 3, 370-381.

15. Sarkar A, Horne DS \& Singh H (2010) Interactions of milk protein-stabilized oil-in-water emulsions with bile salts in a simulated upper intestinal model. Food Hydrocolloids 24, 142-151.

16. Sek L, Porter CJ, Kaukonen AM, et al. (2002) Evaluation of the in-vitro digestion profiles of long and medium chain glycerides and the phase behaviour of their lipolytic products. J Pharm Pharmacol 54, 29-41.

17. Golding M, Wooster TJ, Day L, et al. (2011) Impact of gastric structuring on the lipolysis of emulsified lipids. Soft Matter $\mathbf{7}$, 3513-3523.

18. Day L, Golding M, Xu M, et al. (2014) Tailoring the digestion of structured emulsions using mixed monoglyceride-caseinate interfaces. Food Hydrocolloids 36, 151-161.

19. Marciani L (2011) Assessment of gastrointestinal motor functions by MRI: a comprehensive review. Neurogastroenterol Motil 23, 399-407. 
20. Schwizer W, Steingoetter A \& Fox M (2006) Magnetic resonance imaging for the assessment of gastrointestinal function. Scand J Gastroenterol 41, 1245-1260.

21. Marciani L, Wickham MSJ, Bush D, et al. (2006) Magnetic resonance imaging of the behaviour of oil-in-water emulsions in the gastric lumen of man. Br J Nutr 95, 331-339.

22. Hussein MO, Hoad CL, Wright J, et al. (2015) Fat emulsion intragastric stability and droplet size modulate gastrointestinal responses and subsequent food intake in young adults. J Nutr 145, 1170-1177.

23. Marciani L, Wickham M, Singh G, et al. (2007) Enhancement of intragastric acid stability of a fat emulsion meal delays gastric emptying and increases cholecystokinin release and gallbladder contraction. Am J Physiol Gastrointest Liver Physiol 292, G1607-G1613.

24. Marciani L, Ramanathan C, Tyler DJ, et al. (2001) Fat emulsification measured using NMR transverse relaxation. J Magn Reson 153, 1-6.

25. Musse M, Quellec S, Devaux MF, et al. (2009) An investigation of the structural aspects of the tomato fruit by means of quantitative nuclear magnetic resonance imaging. Magn Reson Imaging 27, 709-719.

26. Mariette F (2009) Investigations of food colloids by NMR and MRI. Curr Opin Colloid Interface Sci 14, 203-211.

27. Le Feunteun S \& Mariette F (2008) Effects of acidification with and without rennet on a concentrated casein system: a kinetic NMR probe diffusion study. Macromolecules $\mathbf{4 1}$, 2079-2086.

28. Curcic J, Sauter M, Schwizer W, et al. (2015) Validation of a golden angle radial sequence (GOLD) for abdominal T1 mapping during free breathing: demonstrating clinical feasibility for quantifying gastric secretion and emptying. J Magn Reson Imaging 41, 157-164.

29. Sauter M, Curcic J, Menne D, et al. (2012) Measuring the interaction of meal and gastric secretion: a combined quantitative magnetic resonance imaging and pharmacokinetic modeling approach. Neurogastroenterol Motil 24, 632-638 e272-e633.
30. Marciani L, Gowland PA, Spiller RC, et al. (2001) Effect of meal viscosity and nutrients on satiety, intragastric dilution, and emptying assessed by MRI. Am J Physiol Gastrointest Liver Physiol 280, G1227-G1233.

31. Hoad CL, Parker H, Hudders N, et al. (2015) Measurement of gastric meal and secretion volumes using magnetic resonance imaging. Phys Med Biol 60, 1367-1383.

32. Reeder SB, McKenzie CA, Pineda AR, et al. (2007) Water-fat separation with IDEAL gradient-echo imaging. J Magn Reson Imaging 25, 644-652.

33. Yu H, McKenzie CA, Shimakawa A, et al. (2007) Multiecho reconstruction for simultaneous water-fat decomposition and T2* estimation. J Magn Reson Imaging 26, 1153-1161.

34. Yu H, Shimakawa A, McKenzie CA, et al. (2008) Multiecho water-fat separation and simultaneous $\mathrm{R} 2 *$ estimation with multifrequency fat spectrum modeling. Magn Reson Med 60, $1122-1134$.

35. Tsao J \& Jiang Y (2013) Hierarchical IDEAL: fast, robust, and multiresolution separation of multiple chemical species from multiple echo times. Magn Reson Med 70, 155-159.

36. Steingoetter A, Fox M, Treier R, et al. (2006) Effects of posture on the physiology of gastric emptying: a magnetic resonance imaging study. Scand J Gastroenterol 41, 1155-1164.

37. Lin LI (1989) A concordance correlation coefficient to evaluate reproducibility. Biometrics 45, 255-268.

38. Marciani L, Wickham M, Hills BP, et al. (2004) Intragastric oilin-water emulsion fat fraction measured using inversion recovery echo-planar magnetic resonance imaging. J Food Sci 69, E290-E296.

39. Banerjee S, Dixit S, Fox M, et al. (2015) Validation of a rapid, semiautomatic image analysis tool for measurement of gastric accommodation and emptying by magnetic resonance imaging. Am J Physiol Gastrointest Liver Physiol 308, G652-G663.

40. R Development Core Team (2015) R: A Language and Environment for Statistical Computing. Vienna: R Foundation for Statistical Computing.

41. Tsao J \& Kozerke S (2012) MRI temporal acceleration techniques. J Magn Reson Imaging 36, 543-560. 